\title{
A Review on Gender Differences in Schizophrenia in Indian Settings
}

\author{
Aishwarya Marathe and Rekha Wagani* \\ Department of Psychology, Amity Institute of Behaviour and Allied Sciences, Amity University Mumbai, India \\ *Corresponding author: Rekha Wagani, Department of Psychology, Amity Institute of Behaviour and Allied Sciences, Amity University \\ Mumbai, India
}

\begin{abstract}
The present article is a scientific review aimed to explore the various gender differences, which are seen in individuals with schizophrenia. Cultural difference is seen as one of the factors playing a major role in the gender differences of people with schizophrenia. The present article presents a comprehensive review of the research done in the past in the area of schizophrenia, thereby presenting a significant summary of the previous research findings. This article focusses on the researches on gender differences in Schizophrenia in Indian context as well as worldwide. It suggests that there are various factors, which have not been further explored, and there is a scope for research in the Indian context, thereby helping to fill in the gaps in the literature related to the factors involved in contributing to the gender differences in Schizophrenia.
\end{abstract}

Keywords: Schizophrenia; Gender Differences; Indian context; Worldwide

\section{Introduction}

Schizophrenia is one of the widely studied disorders across the globe. It is the most widely researched topic by scholars worldwide. It is a chronic and disabling mental illness affecting millions of people worldwide [1-5]. The annual prevalence of Schizophrenia worldwide is $0.2-0.4 / 1000$ [6]. The prevalence of Schizophrenia in India is $3 / 1000$ [7]. The annual incidence rates obtained were 4.4 and 3.8 per 10,000 for rural and urban areas, respectively. There are many gender differences observed in the individuals with Schizophrenia. Men tend to show more Negative symptoms as compared to women who display more of Affective symptoms [8]. Gender differences in terms of prognosis is noted, where women have better outcomes in terms of clinical course and occupational and social functioning [9-11]. Disability being one area where gender differences are evidently seen, men face disability in occupational functioning [12] whereas women in the marital functioning [13]. The cumulative lifetime risk is the same in both the genders [14].

\section{Prevalance and Incidence}

Sex differences in the incidence and prevalence of Schizophrenia may be dependent on the stringency of diagnostic criteria applied. When the diagnostic criteria are broader, there sex differences are less significant [15].

\section{Age of Onset}

Majority of studies done in this area suggests that men have an earlier age at their 1st hospitalisation as compared to woman $[16,17]$. They further noted that these differences in the age of 1st hospitalisation have ranged from 2-6 years. Another research suggests that there has been a general shift in the age of onset between both genders, with women being at a higher rate to have a later onset of symptoms regardless of when hospitalisation first occurred [18]. Castle and [3,8] did a prospective study where they go the average onset of males, which was 31.2 , was almost 10 years younger than the average onset age for females, which was 41.1. In addition, when family ratings of then1st appearance of Schizophrenic symptoms was used, it was clearly seen that men show an earlier onset of schizophrenic symptoms than women $[7,10]$. This difference in the age could also be affected by the familial values versus non-familial status. Albus and [5] conducted a study to see the differences in the age of onset related to familial and nonfamilial status and found that there is no difference in onset age in familial cases of schizophrenia (38 Male-male pairs and 29 femalefemale pairs). It was also evidently seen that the age of onset of earlier for males in non-familial cases. It is widely seen that females have a much later onset of Schizophrenia. Researchers suggest that the females also have a better course of illness than males. They 
suggest that these two phenomena are related to one another, I.e., worse subtype of illness occurs earlier and hence results in later onset in women results in a less aggressive illness thereby resulting into a better outcome. These views are encouraged by epidemiological literature to s great extent [6].

\section{Premorbid Functioning}

Andia [3] did a research on the sex differences in the premorbid functioning in individual with schizophrenia. It was found that females had a higher level of functioning which majorly included greater educational attainment. It was also evidently found that females had a greater likelihood of getting married prior to their onset of illness. There is a positive correlation between premorbid functioning and prognosis. Better the premorbid functioning, better is the prognosis for illness. Shtasel, Gur \& Heinberg [14] found gender differences in premorbid functioning being worse in men than women. McGlashan and Bardenstein [5,9]

did a research related to gender differences and found that females had better premorbid functioning and marital adjustment. Symptomatologic characteristics: Gender differences are vividly seen in symptoms and its exhibition. Females are more likely to present with comorbid depression or even anxiety disorders as opposed to males who are more likely to receive a diagnosis for substance abuse or alcohol abuse and difficulties in impulse control during the first psychiatric admission. When the symptoms are expressed, it is seen that men tend to experience more affective flattening and negative symptoms at the time of their 1st episode as compared to females [4]. These results were also supported by the study done by Rachel Willhite in California. In addition, males were found to have more difficulties related to emotions and were found to be impaired on emotional and social withdrawal, blunted affect, poor rapport. Females on the other hand were seen to have severe somatic symptoms.

\section{Course and Outcome}

Alice \& Chue [2] did a study to explore the gender perspective in course of schizophrenia and found that the course was more favourable in women with less smoking and substance abuse. It was also seen that women presented higher rates oh remission, less days of hospitalisation and better response to typical anti psychotics than men. However, research has contrast view related to gender differences in hospitalisation. Haro and his colleagues [1] found that women presented higher risk of hospitalisation than men. Another study done by Usual and colleagues in 2001 reported that number of previous hospitalisation were similar to both men and women. These 3 contradictory results pave way for further investigation in this regard.

\section{Types of Research Done in India}

\section{Prevalence \& Incidence}

The prevalence of schizophrenia in India is observed to be lower as compared to that in the western countries [16]. In addition to this, it was also seen that the prevalence rate in "least developed" countries was significantly lower as compared to emerging and developed countries. One of the factors which might affect this difference would be "under reporting" (Avasthi [5]). Considering the important aspect of the incidence and prevalence rate across gender, various kinds of results have been noted by differences researchers. Rode [3] did a study on 196 individuals having schizophrenia, of which $55.61 \%$ were males and $44.39 \%$ were females. It is interesting to note these sex differences and understand the factors related to it. This area of research remains least explored in Indian context and is in need of further research.

\section{Age of Onset}

Similar to the research from western countries, it was seen that the gender differences do exist in the age of onset of schizophrenia in Indian population too Murthy [8] found that the males had an earlier onset of the illness as compared to that of females. The males were seen to develop it 5 years earlier than the females [12]. But another study done by Gangadhar and colleagues found contradictory results. It was seen that there was no difference in the onset age between the genders. Although, it was further seen that the proportion of females was higher in group if under 20 years of age. Janakiramiah [11] said that this could be due the over representation of younger ages. Murthy and colleagues in 1998 did a study and found no sex differences in the age of onset. These studies contradict in results thereby paving a way for further research. It is important to find that if the difference in the onset age is really a true onset age or age at case finding. Many factors could affect it and they play a major role. It can be highly possible that the early hospitalisation in men is due to quicker response to symptoms by society rather than early manifestation. It is also possible that the females are cared at home prior to diagnosis which postpones hospitalisation.

\section{Premorbid Functioning}

Stusser [9] reported various types of premorbid abnormalities in individuals with schizophrenia. These abnormalities are exaggerated in intellectual and social areas. Foerster [13] added to these results that such abnormalities I'm the premorbid functioning are seen more in men as compared to women who develop schizophrenia. Other studies also supported the findings where in it was seen that males have higher premorbid abnormalities than females Childers [11]. Retrospective studies reported children seeking mental health services and those at high risk suggest similar results. These premorbid deficits also result in an early onset of illness especially in Male gender. According to the neurodevelopmental model of schizophrenia, the premorbid functioning has abnormal development in early years. Crow [10] confirmed this view adding gender differences related to it. Men have an irritable, disagreeable premorbid functioning. They also tend to be defiant of authority, whereas women tend to be secure and shy and participate less in groups $[1,6]$.

\section{Clinical Representation}

Gender also influences the way the symptoms are expressed. Seeman [1] did a research and found that females are seen to have more affective symptoms and fewer negative symptoms. They also tend to get a diagnosis of schizoaffective disorder. Roberta \& Handel [4] found that females with schizophrenia tend to be more 
hostile as compared to males. They also are physically active and dominating, with more sexual delusions. They tend to be more emotional than men. McGlashan \& Bardenstein [6] did a study and results were consistent with earlier findings that women experience affective symptoms and less negative symptoms. They also said that females exhibit more or anxiety and paranoid symptoms. The meaning of symptoms seems differ across genders and manifest in different ways. Symptoms of withdrawal and dependency reflect as depression syndrome in women whereas negative symptoms in men.

\section{Course and Outcome}

Thara [1] did a longitudinal study of 25 years in madras and found that there were no differences in genders in the course and outcome of schizophrenia. These results contradict the results from western countries. Although, there have been studies which support that women have favourable outcomes than men. Dutta \& Kapur [7] did a research related to course and outcome of schizophrenia and the gender differences in it. They looked at the prognostic factors and found a negative correlation between chances of improvement and duration of illness. Shorted the duration of illness (less than one year), higher are the chances of improvement. Other factors playing important role in improvement are no family history of mental illness, acute onset and younger age. It was also seen that gender and previous history of mental illness had no prognostic significance.

\section{Cultural Influences}

"Culture" plays a very important role in the entire journey of schizophrenia especially in the Indian context. Various studies focus on the culture and its influence on overall functioning of individuals with schizophrenia. Loganathan and Murthy did a research on gender differences and doing marriage, job and children as most important factors being affected. Women had a ear if rejection and were not comfortable disclosing about their illness to their husbands. They used concealing as a strategy. Females also preferred to stay unmarried. It was seen that separation was a common event occurring when told about their illness. Women who expecting were forced to abort their child, and of given birth, were separated from child. On the other hand, men with schizophrenia, faced a lot of frustration in securing jobs. They were highly stigmatised. It was therefore seen that women were separated or divorced while more men remained single.

\section{Lacunae in Researches Today}

The "culture" as a factor which broadly influences the expression of symptoms across genders needs to be investigated further. This investigation may help to identify the unexplored link between the illness and related factors. In the Indian context, religious influences need to be investigated further. To determine various causal mechanisms across the genders, longitudinal studies need to be done.

\section{Studies Required in Indian Setting}

The prevalence and incidence of schizophrenia is seen more in men in India. But the reasons for this higher proportion in one gender remains unexplored. Ignorance and underreporting could be some factors that can be studied in Indian context. Factors involved in favourable outcomes for women in Indian context can be studied further Another area which needs to be explored are the religious influences on the overall course of schizophrenia. Studies related to practising spirituality and outcomes of schizophrenia can be done.

\section{Conclusion}

The evidences noted throughout the paper point towards general susceptibility across genders for schizophrenia. Despite of contrary results in various studies, most of the work consistently show that males are more prone towards early onset, have negative symptoms and less favourable outcome towards Schizophrenia in both, Indian as well as western context. However, research done in India has been without much structure and organisation. Wellcoordinated studies are needed to arrive at clear, structured and organised reports of the gender differences in India. In addition, the results can also be used effectively in planning for gender-sensitive mental health services in India.

\section{References}

1. Aleman A, Kahn RS, Selten JP (2003) Sex differences in the risk of schizophrenia: Evidence from meta-analysis. Arch Gen Psychiatry 60(6): 565-571.

2. Avasthi A (2012) Indianizing psychiatry-Is there a case enough? Indian J Psychiatry 53: 111-120.

3. Castle DJ, Abel KM, Takei N, Murray RM (1995) Gender differences in schizophrenia: Hormonal effect or subtypes. Schizophrenia Bulletin 21(1): 1-12.

4. D L Shtasel, Gur RE, Gallacher C, heimberg, Gur RC (1992) “Gender differences in the clinical expression of schizophrenia. Schizophrenia Research 7(3): 225-231.

5. Gupta S, Kulhara P (2010) What is schizophrenia: A neurodevelopmental or neurodegenerative disorder or a combination of both? A critical analysis. Indian J Psychiatry 52(1): 21-27.

6. Häfner H (1988) Europian Achieve Psychiatry Neurological Science pp: 238-263.

7. Kulhara P, Shah R, Aarya KR (2010) An overview of Indian research in schizophrenia. Indian J Psychiatry 52: S159-S172.

8. Math SB, Chandrashekar CR, Bhugra D (2007) Psychiatric epidemiology in India. Indian J Med Res 126(3): 183-192.

9. McGrath J, Saha S, Welham J (2004) A systematic review of the incidence of schizophrenia: the distribution of rates and the influence of sex, urbanicity, migrant status and methodology. BMC Med 28(2): 13.

10. Mccue RE, Waheed R, Urcuo L (2003) Poly pharmacy in patients with schizophrenia. J Clin Psychiatry 64(9): 984-999.

11. Moriarty PJ, Lieber D, Bennett A, White L, Parrella M, et al. (2001) Gender Differences in Poor Outcome Patients With Lifelong Schizophrenia. Schizophrenia Bulletin 27(1): 103-113.

12. Ochoa S, Usall J, Cobo J, Labad X, Kulkarni J (2012) Gender differences in schizophrenia and first-episode psychosis: a comprehensive literature review. Schizophr Res Treatment pp-916198.

13. Ramdas S, Kuttichira P, Sumesh TP, Ummer SA (2010) A study of an antipsychotic prescription pattern of patients with schizophrenia in a developing country. Indian Journal of Psychology Medicine 32(1): 13-16.

14. Rangaswamy T (2012) Twenty-five years of schizophrenia: The Madras longitudinal study. Indian J Psychiatry 54(2): 134-137. 
15. Rode SB, Salankar HV, Verma PR, Sinha U, Ajagallay RK (2014) Pharmacoepidemiological survey of schizophrenia in Central India. International Journal Research Medical Science 2(3): 1058-1062.

16. McGlashan TH, Bardenstein KK (1990) Gender differences in affective, schizoaffective, and schizophrenic disorders. Schizophrenia Bulletin 16(2): 319-329.

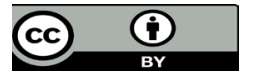

This work is licensed under Creative Commons Attribution 4.0 License

To Submit Your Article Click Here:

Submit Article

DOI: $10.32474 /$ SJPBS.2019.03.000158
17. Trivedi JK, Dhyani M, Yadav VS, Rai SB, Sinha PK (2010) Anti-psychotic drug prescription pattern for schizophrenia: an Indian perspective. Indian J Psychiatry 52(3): 279-316.

18. Thara R, Kamath S (2015) Women and schizophrenia. Indian journal of psychiatry 57(6): 246-251.

\begin{tabular}{|c|c|}
\hline SJ & $\begin{array}{l}\text { Scholarly Journal of Psychology } \\
\text { and Behavioral Sciences }\end{array}$ \\
\hline & $\begin{array}{l}\text { Assets of Publishing with us } \\
\text { - Global archiving of articles } \\
\text { - Immediate, unrestricted online access } \\
\text { - Rigorous Peer Review Process }\end{array}$ \\
\hline $\begin{array}{c}\text { Scholarly Journal of } \\
\text { Psychology \& Behavioral sciences }\end{array}$ & $\begin{array}{l}\text { - Authors Retain Copyrights } \\
\text { - Unique DOI for all articles }\end{array}$ \\
\hline
\end{tabular}

